\title{
Subclinical TRAPS treated with canakinumab
}

\author{
M. Amatruda, N.S. Carucci, C. Fede, G. Conti \\ Pediatric Nephrology and Rheumatology Unit with Dialysis, Department of Human Pathology in Adulthood \\ and Childhood "G. Barresi"; AOU Policlinic "G. Martino", Messina University, Italy
}

\begin{abstract}
SUMMARY
Tumor necrosis factor receptor-associated periodic syndrome (TRAPS) is a rare autoinflammatory disease characterized by recurrent episodes of fever and systemic inflammation. We describe the case of a 19-year-old patient who was referred to our attention with frequent subclinical TRAPS episodes characterized by mild arthralgias and crampy abdominal pain, without fever. Inflammatory markers, including serum amyloid A which increases the risk of long-term amyloidosis, were persistently high also when the patient was in good general conditions. Therapy with human anti-interleukin $1 \beta$ monoclonal antibody, canakinumab, led to disease control and normalization of the inflammatory markers, which are currently still normal. This clinical case supports the need to treat also subclinical TRAPS. In this respect, canakinumab is effective and reduces the risk of developing amyloidosis.
\end{abstract}

Key words: TRAPS; IL-1, canakinumab, AA amyloidosis.

\section{INTRODUCTION}

T umor necrosis factor (TNF) receptor-associated periodic syndrome (TRAPS) is a rare autosomal-dominant autoinflammatory disease characterized by an inappropriate activity of the innate immune system caused by mutations in the gene for the TNF receptor superfamily member 1A (TNFRSF1A) $(1,2)$. TRAPS diagnosis is based upon a family medical history and thorough clinical evaluation. TRAPS is characterized by recurrent and long-lasting (more than 7 days) fever associated with other variable symptoms: myalgias, arthralgia, centrifugal migratory erythematous rash, chest and/or abdominal pain accompanied by nausea, vomiting, diarrhea or constipation and, finally, ocular manifestations (conjunctivitis and periorbital oedema). Secondary amyloidosis is the most serious long-term complication of TRAPS and, when affecting the kidney, often leads to rapid deterioration of renal function that manifests with proteinuria and kidney failure. Clinical suspicion of TRAPS is confirmed by molecular genetic testing, which can provide evidence of mutations in the TNFRSF1A gene (1-3).
Recent studies have confirmed the efficacy of canakinumab in this condition. It is a human monoclonal anti-interleukin $1 \beta$ (IL$1 \beta)$ antibody, which can induce a rapid and complete remission in patients with active TRAPS $(4,5)$. IL- $1 \beta$ is a proinflammatory cytokine that plays a key role in the activation of the innate immune system and response in the acute phase. Its importance has been demonstrated in studies of cryopyrinassociated periodic syndrome (CAPS), a rare inherited autoinflammatory disorder. The incredibly swift resolution of symptoms with IL-1 $\beta$ blockade has suggested that this may be a valuable approach to the management of other inherited autoinflammatory disorders, such as TRAPS (6). In fact, not only anti-IL-1 $\beta$ has recently proven to control recurrent TRAPS episodes, but also to reduce the prolonged acute-phase response and deposition of serum amyloid A. Hence, in 2016 the US Food and Drug Administration approved canakinumab for the treatment of TRAPS (4).

To our knowledge there are no studies or evidence on the use of canakinumab in patients with subclinical TRAPS episodes, characterized by mild symptoms without fever. The aim of this case report is to sup- $\overline{\text { Corresponding author: }}$ Giovanni Conti

Pediatric Nephrology and Rheumatology Unit with Dialysis, Department of Human Pathology in Adulthood and Childhood "G. Barresi"; AOU Policlinic "G. Martino" Messina Strada Consolare Valeria, 98124 Messina, Italy E-mail: giovanniconti@hotmail.com 
port the need for early treatment of patients with milder form of TRAPS, especially if there are constantly high C-reactive protein (CRP) and serum amyloid A (SAA) levels, in order to reduce the underlying inflammatory state and prevent the risk of developing complications, such as amyloidosis, over time. This clinical case shows that the treatment with long-lasting IL-1 $\beta$ blocker, canakinumab, is effective in the control of the chronic inflammatory manifestations and can prevent the possible development of long-term complications.

\section{CASE REPORT}

We describe the case of a 19-year-old patient with a diagnosis of TRAPS and a heterozygous T50M missense mutation in the TNFRSF1A gene. There was nothing relevant in the family and personal medical histories of the patient.
At the age of 7, he started to present frequent and periodic episodes of fever accompanied by crampy mild abdominal pain, arthralgias and sometimes nausea, vomiting and red eyes. During these attacks, that occurred cyclically every 3-4 months, the laboratory tests showed neutrophilic leukocytosis and increase of acute phase inflammatory markers, such as CRP and erythrocyte sedimentation rate (ERS). When the patient was in good general conditions, the SAA level was normal. A diagnosis of TRAPS was made. In several occasions during the attacks, he was treated with glucocorticoids (prednisone $1 \mathrm{mg} /$ $\mathrm{kg}$ /day for 5-7 days) or non-steroidal antiinflammatory drugs (NSAIDs) therapy and subsequently recovered.

At the age of 15, eight years after the diagnosis of TRAPS, he continued to present frequent attacks of mild abdominal pain and lower limb arthralgia, but in the

Table I - Monitoring of laboratory tests before and after starting treatment with canakinumab.

\begin{tabular}{|c|c|c|c|c|c|c|c|c|c|c|}
\hline & $\begin{array}{c}\begin{array}{c}\text { Normal } \\
\text { values }\end{array} \\
\end{array}$ & 2015 & 2016 & 2017 & $\begin{array}{c}\text { December } 2018 \\
\text { before anti-IL-1B }\end{array}$ & $\begin{array}{c}\text { January } 2019 \\
\text { after anti-IL-1B }\end{array}$ & $\begin{array}{l}\text { March } \\
2019\end{array}$ & $\begin{array}{l}\text { July } \\
2019\end{array}$ & $\begin{array}{l}\text { October } \\
2019\end{array}$ & $\begin{array}{c}\text { January } \\
2020\end{array}$ \\
\hline $\begin{array}{l}\text { Red blood cell } \\
(\mathrm{mmc})\end{array}$ & $\begin{array}{l}4.5-5.5 \\
\times 10^{6}\end{array}$ & $5.4 \times 10^{6}$ & $5.4 \times 10^{6}$ & $5.9 \times 10^{6}$ & $5.2 \times 10^{6}$ & $5.9 \times 10^{6}$ & $6.0 \times 10^{6}$ & $6.0 \times 10^{6}$ & $6.2 \times 10^{6}$ & $6.1 \times 10^{6}$ \\
\hline $\begin{array}{l}\text { Hemoglobin } \\
\text { (gr\%) }\end{array}$ & $14-17$ & 13.4 & 14.3 & 15.2 & 14.6 & 16.0 & 16.6 & 16.7 & 18.1 & 17.0 \\
\hline $\begin{array}{l}\text { White blood } \\
\text { cells (mmc) }\end{array}$ & $5-10 \times 10^{3}$ & $12 \times 10^{3}$ & $13 \times 10^{3}$ & $7.8 \times 10^{3}$ & $12.5 \times 10^{3}$ & $5.4 \times 10^{3}$ & $7.8 \times 10^{3}$ & $6.9 \times 10^{3}$ & $5.9 \times 10^{3}$ & $11.2 \times 10^{3}$ \\
\hline $\begin{array}{l}\text { Neutrophils } \\
(\%)\end{array}$ & $54-62$ & 72 & 71 & 57 & 75 & 40 & 54 & 43 & 53 & 69 \\
\hline $\begin{array}{l}\text { Lymphocytes } \\
(\%)\end{array}$ & $25-33$ & 19 & 20 & 35 & 20 & 54 & 38 & 46 & 38 & 19 \\
\hline $\begin{array}{l}\text { Platelets } \\
\text { (mmc) }\end{array}$ & \begin{tabular}{|c|}
$140-400$ \\
$\times 10^{3}$
\end{tabular} & $224 \times 10^{3}$ & $268 \times 10^{3}$ & $297 \times 10^{3}$ & $292 \times 10^{3}$ & $207 \times 10^{3}$ & $153 \times 10^{3}$ & $155 \times 10^{3}$ & $152 \times 10^{3}$ & $172 \times 10^{3}$ \\
\hline $\begin{array}{l}\text { C-reactive } \\
\text { protein } \\
(\mathrm{mg} / \mathrm{dL})\end{array}$ & $<1$ & 15.9 & 18.2 & 3.4 & 8.5 & $<1$ & $<1$ & $<1$ & $<1$ & $<1$ \\
\hline $\begin{array}{l}\text { Erythrocyte } \\
\text { sedimentation } \\
\text { rate }(\mathrm{mm} / \mathrm{h})\end{array}$ & $<20$ & 40 & 46 & 23 & 38 & $<20$ & $<20$ & $<20$ & $<20$ & $<20$ \\
\hline $\begin{array}{l}\text { Serum } \\
\text { amyloid A } \\
(\mu \mathrm{g} / \mathrm{mL})\end{array}$ & $<10$ & 198.8 & 171.0 & 198.2 & 199.2 & 6.7 & 6.6 & 5.8 & 7.2 & 5.6 \\
\hline $\begin{array}{l}\text { Albumin } \\
\text { urinary } \\
\text { excretion } \\
\text { ( } \mu \mathrm{g} / \mathrm{min})\end{array}$ & $<30$ & 24.5 & 18 & 22.3 & 21.6 & 10.8 & 11.6 & 10.8 & 12.1 & 14.4 \\
\hline
\end{tabular}


absence of fever and without periodicity. The inflammatory markers were persistently high, also when the patient was in good general conditions. Chest radiograph, abdominal ultrasound and fecal calprotectin assay were normal. There was no need for any treatment, because these episodes resolved spontaneously. Three years later, considering the increased risk of long-term amyloidosis and chronic vascular damage due to the persistent inflammatory state and high SAA levels, he started canakinumab ( $4 \mathrm{mg} / \mathrm{kg}$ every 4 weeks). Proteinuria and microalbuminuria were within the normal range. After the treatment, prompt clinical and laboratory remission was achieved, including SAA normalization. Monitoring of laboratory tests is described in Table I.

Today, the patient continues canakinumab treatment with persistent benefits and without side effects.

\section{DISCUSSION AND CONCLUSIONS}

TRAPS is a rare disease, caused by mutations of genes that code for TNFRSF1A, the most potent inflammatory cytokine of innate immunity. P46L and R92Q are the two most common TNFRSF1A variants, which are present in $10 \%$ of West Africans and $2 \%$ of Caucasians, respectively. The vast majority of carriers of these two variants are in good health. It is still unclear how these variants actually cause the inflammatory disease in a minority of patients (2). Although often no trigger is described, TRAPS attacks can be induced by minor trauma, menstrual cycle, fatigue, vaccinations, infections, emotional stress and physical exercise.

In a case series of 158 patients the median age of onset of symptoms was 4.3 years, but $9.1 \%$ of them presented an onset after 30 years of age (2). The clinical phenotype depends on the age of onset. Pediatric onset patients have prolonged attacks, lasting from less than 1 week to 3 weeks, accompanied by a variety of features including recurrent fever and others $(1,2,7)$. These patients tend to have fewer recurring fever episodes during adulthood and to develop a chronic disease characterized by undulating and mild symptoms with persistent increase of acute inflammatory markers. Adult-onset patients have a serous involvement, with higher incidence of pleurisy and pericarditis $(1,7)$. Steroids are useful in terminating attacks, but their effect tends to wane over time. In some cases (arthromyalgia and periorbital edema), NSAIDs provide some degree of symptom relief $(1,2)$. Compared to other periodic fever syndromes (familial Mediterranean fever and mevalonate kinase deficiency), the acute TRAPS attacks last longer and generally occur 2-4 times a year (8). Some patients, like in this case report, have a subclinical state with no fever, only mild symptoms and persistent acute inflammatory response between clinical flares (9). Kallinich et al. (10) described the case of a patient with a family history of TRAPS, who therefore had genetic testing and subsequent counseling. Molecular analysis of the TNFRSF1A gene showed a heterozygous T50M mutation in all family members. Only after this finding, she admitted retrospectively recurrent sore muscles. During follow-up she developed marked proteinuria and a kidney biopsy showed considerable amyloid A (AA) deposition. This case shows how the heterozygous T50M mutation of the TNFRSF1A gene can determine even minimal clinical signs, but at the same time a chronic inflammatory state, as in our patient, at risk of evolving into AA amyloidosis (10). The heterozygous T50M missense mutation is a non-cysteine mutation, but a single base-pair mutation causing a threonine to methionine exchange at amino acid position 50 within the TNFRSF1A gene. Threonine on position 50 causes an intrachain hydrogen bond that changes the receptor conformation and impairs its function. Despite a supposedly benign underlying mutation, high penetrance T50M mutation is often associated with a severe phenotype and a high risk of long-term systemic amyloidosis (11).

SAA is a very sensitive and dynamic major acute-phase protein, mainly produced in the liver under the regulation of IL-1, IL- 6 and TNF- $\alpha$. Its expression was also 
demonstrated in other cell types, including macrophages, endothelial cells and smooth muscle cells (12). If the inflammatory trigger persists, the SAA level may reach a critical threshold over which it becomes prone to aggregation and deposition in tissues and organs, thus developing a secondary acquired disorder, AA amyloidosis (12). This is the riskiest TRAPS complication, which is found in about $18 \%$ of adult, untreated patients (2). The predominant manifestation of AA amyloidosis is renal dysfunction, which, if not appropriately treated, leads to end-stage kidney disease and renal replacement therapy, with a high mortality rate (12). Treatment of AA amyloidosis should be initiated in order to suppress the underlying inflammatory disease and reduce the SAA level as much as possible.

In this case report, canakinumab has been used to treat a patient with a persistent and subclinical inflammatory state, with a consequent increased risk of long-term amyloidosis. We achieved a satisfactory clinical and laboratory response, with a complete remission of the disease in the following months. The inflammatory markers, including the SAA, are still normal after 16 months of therapy. In our opinion, canakinumab has significantly reduced the risk of amyloidosis and renal complication. In conclusion, this case report suggests that it is advisable to pay attention to the TRAPS subclinical symptoms even in the absence of fever, especially if there are constantly high CRP and SAA levels. The disease should be treated early, because the underlying inflammatory state can lead to amyloidosis over time. Based on our experience, canakinumab is effective to reach both clinical and laboratory remission.

Conflicts of interest: the authors declare that they have no conflicts of interest.

\section{REFERENCES}

1. Lachmann HJ. Periodic fever syndromes. Best Pract Res Clin Rheumatol. 2017; 31: 596-609.
2. Lachmann HJ, Papa R, Gerhold K, et al. The phenotype of TNF receptor-associated autoinflammatory syndrome (TRAPS) at presentation: a series of 158 cases from the Eurofever/EUROTRAPS international registry. Ann Rheum Dis. 2014; 73: 2160-2167.

3. Gattorno M, Hofer M, Federici S, et al. Eurofever Registry and the Paediatric Rheumatology International Trials Organisation (PRINTO). Classification criteria for autoinflammatory recurrent fevers. Ann Rheum Dis. 2019; 78: 1025-1032.

4. Gattorno M, Obici L, Cattalini M, et al. Canakinumab treatment for patients with active recurrent or chronic TNF receptor-associated periodic syndrome (TRAPS): an openlabel, phase II study. Ann Rheum Dis. 2017; 76: 173-178.

5. De Benedetti F, Gattorno M, Anton J, et al. Canakinumab for the Treatment of Autoinflammatory Recurrent Fever Syndromes. N Engl J Med. 2018; 378: 1908-1919.

6. Lachmann HJ, Quartier P, So A, Hawkins PN. The emerging role of interleukin- $1 \beta$ in autoinflammatory diseases. Arthritis Rheum. 2011; 63: 314-324.

7. Miyamae T, Hanaya A, Kawamoto M, et al. Diagnostic rate of autoinflammatory diseases evaluated by fever patterns in pediatric- and adult-onset patients. J Clin Rheumatol. 2020; 26: 60-62.

8. Procopio V, Manti S, Bianco G, et al. Genotype-phenotype correlation in FMF patients: A "non classic" recessive autosomal or "atypical" dominant autosomal inheritance? Gene. 2018; 641: 279-286.

9. Quillinan N, Mohammad A, Mannion G, et al. Imaging evidence for persistent subclinical fasciitis and arthritis in tumor necrosis factor receptor-associated periodic syndrome (TRAPS) between febrile attacks. Ann Rheum Dis. 2010; 69: 1408-1409.

10. Kallinich T, Haffner D, Rudolph B, et al. "Periodic fever" without fever: two cases of non-febrile TRAPS with mutations in the TNFRSF1A gene presenting with episodes of inflammation or monosymptomatic amyloidosis. Ann Rheum Dis. 2006; 65: 958-960.

11. Kallinich T, Briese S, Roesler J, et al. Two familial cases with tumor necrosis factor receptor-associated periodic syndrome caused by a non-cysteine mutation (T50M) in the TNFRSF1A gene associated with severe multiorganic amyloidosis. J Rheumatol. 2004; 31 : 2519-2522.

12. Scarpioni R, Ricardi M, Albertazzi V. Secondary amyloidosis in autoinflammatory diseases and the role of inflammation in renal damage. World J Nephrol. 2016; 5: 66-75. 